\title{
FREY SYNDROME COMPLICATING PAROTIDECTOMY: A CASE REPORT AND REVIEW OF LITERATURE.
}

\author{
Adoga AS (FWACS) ${ }^{1}$, John EN (MBBS) ${ }^{1}$, Ma`an $(\mathrm{MBBS})^{1}$ \\ ${ }^{\prime}$ Department of Surgery, Ear, Nose \& Throat Unit, Jos University Teaching Hospital, Jos, Plateau State. \\ Correspondence: Dr Samuel Agida Adoga; Tel: +234(0)8036816636; e-mail: agidadoga@yahoo.com
}

\begin{abstract}
:
The parotid gland is one of the major salivary glands located on the face. It is commonly affected by diseases necessitating its surgical removal. The facial nerve is in intimate contact with this structure and can easily be damaged during operation on this gland. Parotidectomy is the surgical operation done to remove part or the whole of the parotid gland. There are several types of parotidectomies. These may be superficial, total, total conservative, radical or extended radical. A number of complications may ensue following this procedure. Frey syndrome is one of such complications but uncommon. It is therefore the aim of this paper to present this index case with a review ofliterature.
\end{abstract}

Keywords: Frey syndrome, Parotidectomy, Complications

\section{Introduction:}

Parotid gland is one of the major salivary glands located on the lateral aspect of the face. 1 it is affected by several diseases requiring its removal surgically. 2The facial nerve is in intimate contact with this structure and can easily be damaged during operation on this gland.3 Parotidectomy is the surgical operation done to remove part or the whole of the parotid gland. There are several types which comprise the following; superficial, total, total conservative, radical and extended parotidectomies.4Each of these can become complicated. Some of the possible complications are haemorhage, wound infections, salivary gland fistula, facial nerve palsy, recurent disease and Frey's syndrome.5

\section{Case report}

A.R is a 55 year old house wife who presented to the ENT clinic of ECWA-Evangel Hospital, Jos 12 months post parotidectomy with history of sweating and discomfort over the left parotid area any time she sights food. Nine months prior to this, she had a parotidectomy for which she gave history of progressive painless left parotid mass. The swelling was unrelated to sighting food and does not fluctuate in size. No features of facial nerve palsy, no history of drug ingestion like coprozamole, steroids likely to cause parotid swelling, no past history of systemic illnesses like liver diseases, diabetic mellitus or malnutrition. No past history of trauma or irradiation to the region. There was no history suggestive of metastases to the lungs, bones or lower back or the abdomen.

The other systems were essentially normal. She was investigated and a diagnosis of left parotid pleomorphic adenoma was made. She had a left superficial Parotidectomy which was histologically confirmed as pleomorphic adenoma.

Examination revealed a healthy looking middle aged woman, afebrile, not pale.

The facial examination revealed a left cervicomastoidfacial (Lazy-S-incision) over the parotid area. The over lying skin was normal with no facial nerve palsy.

The iodine test was carried out by applying tincture of iodine with radius of $5 \mathrm{~cm}$ over the parotid skin and allowed to dry. She was then given lemon sweet to lick and after two minutes the iodine spot and overlying skin became wet. Starch was then applied over the iodine spot and the overlying skin. Only the iodine spot turned blue black (positive iodine test)

\section{Discussion:}

Frey syndrome comprises sweating and flushing over the parotid region following the sight of food. 6 The incidence is approximately 50 percent of reported cases while other literature puts it as occurring in $90 \%$ post parotidectomy cases if sort for with test.7 The parotid gland is innervated by parasympathetic fibres from the inferior salivatory nucleus to the glossopharyngeal nerve traveling with the auriculotemporal nerve and sympathetic nerve from the superior cervical ganglion of the sympathetic chain which travels along the internal carotid to innervate the gland. The sweat gland within the overlying skin over the parotid gland is innervated by post ganglionic sympathetic fibres also. Stimulation of the parasympathetic nerve supplying the parotid gland leads to increased salivation. Stimulation of the post ganglionic sympathetic fibres supplying the sweat glands in the overlying skin leads to sweating. Parotidectomy leads to transection of some or all of the branches of the post ganglionic parasympathetic nerve fibres to the glands and the sympathetic nerve to the overlying skin. Following this, there is a regeneration of some of this post ganglionic parasympathetic fibres which get united to the post ganglionic sympathetic nerve fibres supplying the overlying skin (misdirection of nerves).Hence when stimulus for salivation comes the final signal is vasodilatation and skin sweating because of this misdirected signal.8,9

The other aetiological factors include congenital causes, any non surgical trauma and irradiation to the parotid area, mandibular fracture, chemotherapy, submandibular gland excision should be sort for in the history. 10, 16, 17

This syndrome was first observed by a woman Lucie Frey in 1923 and she called it Frey syndrome.13 this syndrome does not only affect the parasympathetic fibres of the glossopharyngeal nerve but can affect the auriculotemporal nerve, greater auricular and the lesser occipital nerve.10 Several synonyms have been ascribed such as auriculotemporal syndrome, considered a misnomer because other nerves can be involved.14 The other names are Baillager syndrome, Dupuy syndrome, gustatory 


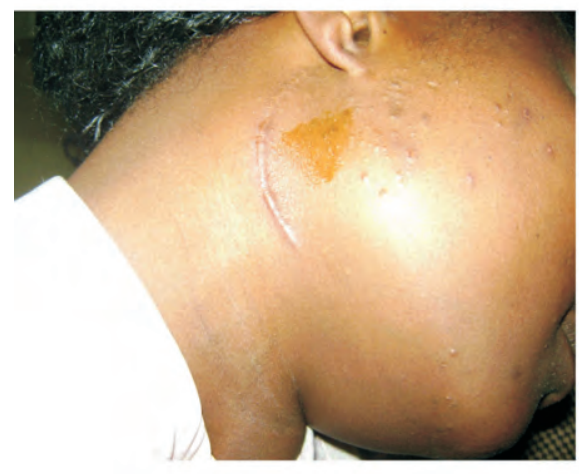

Figure 1: Before the iodine test

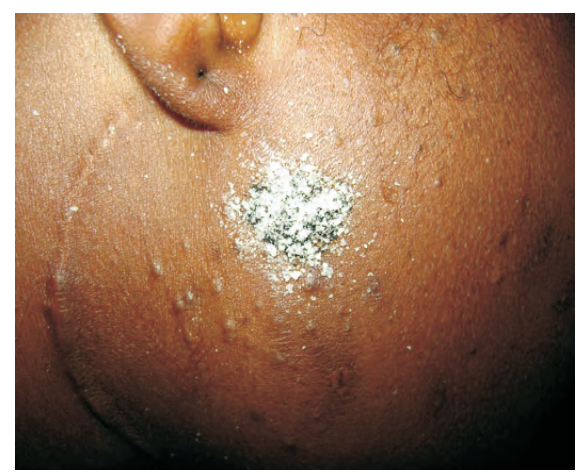

Figure 2: Positive iodine test

syndrome and sudosalivoparous syndrome. 15

The clinical features are that of gustatory sweating, facial flushing with little or no discomfort. There is usually a history of surgery.16 Our patient presented with facial sweating and mild facial discomfort. The other systems are usually normal

The simplest investigation is iodine test and very simple to do.17 In our case it was positive.

The treatment varies depending on severity of symptoms. It may be reassurance, medical or surgical. Usually reassurance is all that is needed because in most cases it is mere discomfort.

Medical treatment includes use of anticholinergic drug like $3 \%$ glycopyrolate application or scopolamine ointment, Use of botulinion toxin. The surgical options are the use of superficial musculoaponeurotic systems (SMAS) to bridge the gap between the parotid surface and the skin may be used as prophylaxis or for treatment. The others are neurectomies i.e. chorda neurectomy or intratympanic parasympatoneurectomy.In our case the symptoms are very mild and patient is doing very well with reassurance. 15,18 , 19

In conclusion, Frey syndrome though reported to have a high incidence in various literature searches; this is the first reported case in our environment and did not require any aggressive surgical intervention.

\section{References:}

1. Wilhemi BJ, Mowlavi A, Neumeister MW.The safe face lift with bony land marks to elevate the SMAS.Plast Reconstr Surg.2003;111.:1723.
2. Saylor AM, Bova R, Coman WB. Parotidectomy- A Clinical Review of Treatment and Outcome at the Princess 